Gut, 1985, 26, 999-1003

\title{
Crypt cell production rate in ulcerative proctocolitis: differential increments in remission and relapse
}

\author{
A ALLAN, J B BRISTOL, AND R C N WILLIAMSON \\ From the University Department of Surgery, Bristol Royal Infirmary, Bristol
}

SUMmARY Crypt cell production rate (CCPR) has been measured by a stathmokinetic technique in the relapse and remission phases of ulcerative proctocolitis. Rectal biopsies were obtained from eight patients with colitis in relapse, 14 patients with colitis in remission, and 14 patients with histologically normal mucosa. Biopsies were maintained in organ culture for 16 hours and were then exposed to vincristine for one to three hours. Crypt cell production rate was determined from the rate of accumulation of arrested metaphases. Mean CCPR in the relapse group (14.2 cells/crypt/hour) was $45 \%$ faster than in the remission group $(9.8 \mathrm{cells} / \mathrm{crypt} / \mathrm{hour} ; \mathrm{p}<0.001)$, which was in turn $14 \%$ faster than in normal mucosa $(8.6$ cells/crypt/hour; $p<0.04)$. More rapid turnover of the rectal epithelium in quiescent as well as active colitis may help to explain the enhanced risk of carcinogenesis in this disease.

Cytokinetic changes in the large bowel mucosa in ulcerative proctocolitis must surely be relevant to the increased risk of colorectal carcinoma in these patients. Early estimations of the mitotic index in rectal biopsies suggested a prolonged cell turnover time in active disease. ${ }^{12}$ By contrast, subsequent and more sophisticated studies of biopsy material have shown a shorter turnover time, an increased labelling index and an enlarged proliferative compartment in the crypt. ${ }^{3-5}$ Because patients with colitis may spend longer in remission than relapse, the proliferative status of quiescent mucosa could be of crucial importance in determining the predisposition to cancer. Here again there is controversy: some authors report that cell proliferation in colitis in remission is not significantly different from normal, ${ }^{12}$ others that it is increased..$^{5}$

All these studies have involved measuring the number of cells in a single phase ( $\mathrm{M}$ or $\mathrm{S}$ ) of the cell cycle. This index is static and may not allow for certain important variables, such as changes in crypt size or cell cycle time. ${ }^{6}$ The crypt cell production rate (CCPR), estimated by plotting the rate of accumulated metaphases in individual crypts, offers a sensitive, dynamic and precise determinant of intestinal cell proliferation, ${ }^{6}$ but methodological and ethical constraints have largely confined its applica-

Address for correspondence: Professor R C N Williamson. University Department of Surgery. Bristol Royal Infirmary. Bristol BS2 8HW.

Received for publication 15 November 1984 tion to animal studies. By adapting the technique to study human rectal biopsies maintained in organ culture, we have been able to measure cell proliferation in whole crypts for the first time in man. We have found an increased CCPR in patients with ulcerative proctocolitis both in remission and relapse.

\section{Methods}

\section{PATIENTS}

Rectal biopsies were taken from seven men and seven women with histologically normal mucosa (mean age 51 years, range 24-79 years). Five each of these controls had haemorrhoids or previous colonic neoplasia (without overt recurrence), and one each had anal fissure, anal fistula, diverticular disease and irritable bowel syndrome. Rectal biopsies were also taken from eight men and six women with ulcerative colitis in histological remission (mean age 55 years, range 33-75 years), and from four men and four women with ulcerative colitis in histological relapse (mean age 40 years, range 14-64 years). The mean duration of disease in the remission group (11.7 years, range $1.5-19$ years) was similar to that in the relapse group ( $7 \cdot 2$ years, range $1 \cdot 5-14$ years). Nineteen of the 22 patients with colitis were receiving oral salazopyrine, and in addition four of the patients in relapse were receiving prednisolone by mouth $(n=3)$ or as a retention enema $(n=2)$. The project was accepted by the district ethical commit- 
tee, and informed consent was obtained from each patient.

Two adjacent mucosal biopsies were obtained at a point $10 \mathrm{~cm}$ from the anal margin. One biopsy was placed in $10 \%$ formalin for subsequent histological classification, while the other was used for organ culture. From the formalin fixed specimens, $4 \mu \mathrm{m}$ sections were cut from paraffin blocks and stained with haematoxylin and eosin.

Rectal biopsies in histological relapse were subdivided according to the scheme of Truelove and Richards. ${ }^{7}$ Biopsies exhibiting irregular crypts, with oedema, vascular congestion and interstitial haemorrhage of the lamina propria, were defined as class I. The epithelium in these biopsies was generally intact, and inflammatory infiltration of the lamina propria was mild. Those biopsies with more severe inflammatory changes constituted class II. Histological assessment was carried out by a pathologist unaware of the findings of the CCPR experiments. Transmission electronmicroscopy was undertaken in addition to conventional examination. Tissue specimens were embedded in epoxy resin, and ultrathin sections were prepared and stained with lead citrate before viewing in a Phillips EM201.

Rectal mucosa was maintained in organ culture for 16 hours according to the method previously described. ${ }^{8}$ In brief, biopsies were cultured in organ culture dishes over RPMI 1640 culture medium (Flow Labs, Ltd) with $10 \% \mathrm{v} / \mathrm{v}$ fetal calf serum. The dishes were sealed in an atmosphere of $95 \% \mathrm{O}_{2}: 5 \%$ $\mathrm{CO}_{2}$, and the temperature was maintained at $37^{\circ} \mathrm{C}$. After 16 hours the culture medium was changed, and vincristine-containing medium was added to the culture dishes. Preliminary experiments established that the optimum dose of vincristine required to produce complete metaphase arrest was $0.5 \mu \mathrm{g} / \mathrm{ml}$ culture medium for normal mucosa and colitis in remission but $1.0 \mu \mathrm{g} / \mathrm{ml}$ for colitis in relapse. Two samples of mucosa were removed from the dishes at 60,120 , and 180 minute intervals after exposure to vincristine. Samples were fixed in Carnoy's solution for 60 minutes and preserved in $70 \%$ alcohol. Later, biopsies were rehydrated by immersion for successive three minute periods in $50 \%$ then $25 \%$ alcohol. After hydrolysis for six minutes in $1 \mathrm{M} \mathrm{HCL}$ at $60^{\circ} \mathrm{C}$, specimens were stained with fresh Schiff's reagent. Ten individual crypts were microdissected from each mucosal specimen and squashed under a cover slip. Metaphase arrest figures were counted under magnification. The mean number of arrested metaphases per specimen was plotted at each time point, and the slope of the resultant line was determined by linear regression analysis (least squares). This figure represents the rate of accumulation of arrested metaphases per minute and when multiplied by 60 gives the CCPR in cells/crypt/hour. Statistical analysis of differences in CCPR between groups of patients was undertaken using Student's $t$-test.

\section{Results}

In a preliminary experiment, linear accumulation of metaphase figures was observed in rectal biopsies exposed to vincristine between 16-19 hours of organ culture. There was a close correlation between metaphase arrest and time $(r=0.99 ; p<0.001)$ (Fig 1). Transmission electronmicroscopy revealed virtually normal preservation of histological architecture in biopsies after 19 hours of organ culture (Figs $2 a, b, c)$.

In 14 patients with histologically normal mucosa CCPR was $8.6 \pm 0.4$ cells/crypt/hour (mean \pm s.e.m.). In 14 patients with colitis in remission CCPR was $9.8 \pm 0.5 \mathrm{cells} / \mathrm{crypt} / \mathrm{hour}$, a $14 \%$ increase over control values $(p<0.04)$. In eight patients with colitis in relapse CCPR was $14 \cdot 2 \pm 0.8$ cells/crypt/hour; this value is $45 \%$ greater than for colitis in remission $(p<0.001)$ and $65 \%$ greater than for controls $(p<0.001)$. Among patients with active colitis, five with mild to moderate inflammation (class I) had a mean CCPR of $14 \cdot 1$ (range 12.6-16.1) and three with severe inflammation (class II) had a mean CCPR of 14.6 (range 12.4-16.7). None of the patients had histological evidence of dysplasia on rectal biopsy. Minor degrees of dysplasia are difficult to assess in the presence of active inflammation and changes are often patchy. ${ }^{9}$ Crypt cell production rate was unrelated to sex, age, drug therapy or duration of disease.

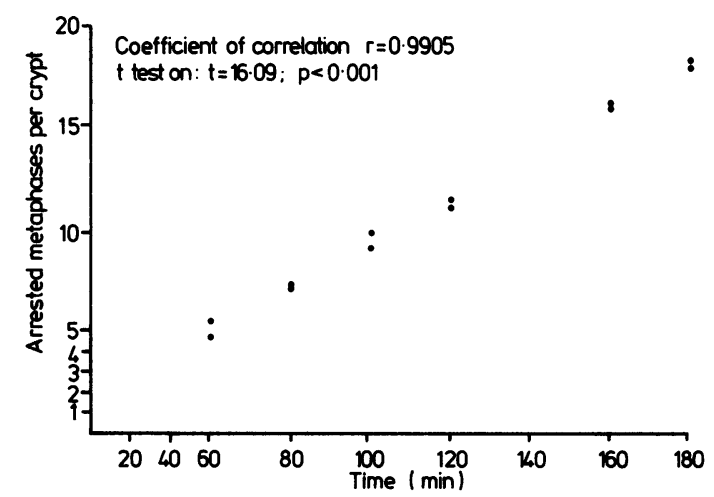

Fig. 1 Accumulation of metaphase arrest figures over the period of assessment. Points represent the mean number of arrested figures in 10 crypts. Two samples were analysed at each time point. 

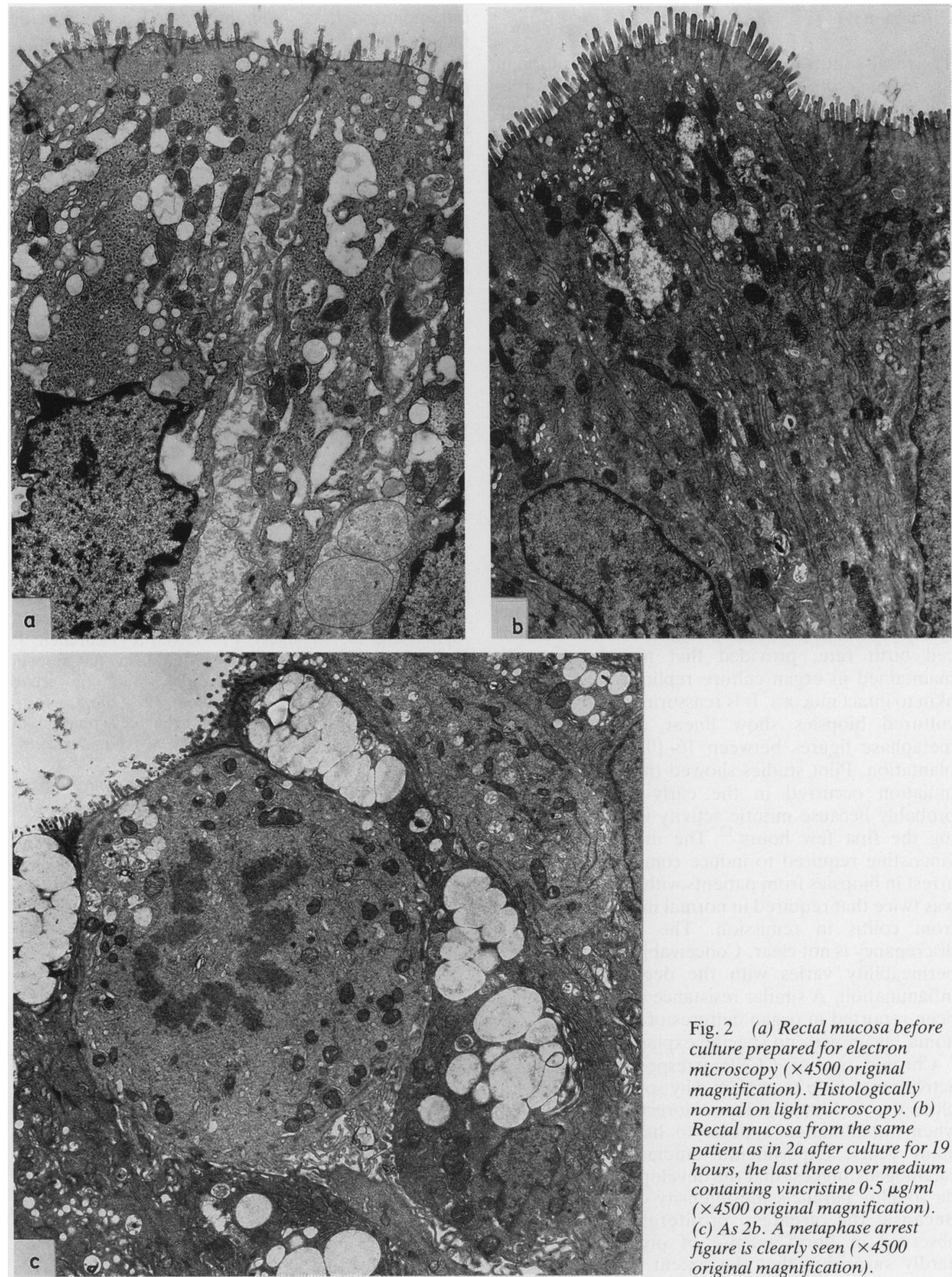

Fig. 2 (a) Rectal mucosa before culture prepared for electron microscopy $(\times 4500$ original magnification). Histologically normal on light microscopy. (b) Rectal mucosa from the same patient as in $2 a$ after culture for 19 hours, the last three over medium containing vincristine $0.5 \mu \mathrm{g} / \mathrm{ml}$ ( $\times 4500$ original magnification). (c) As 2b. A metaphase arrest figure is clearly seen $(\times 4500$ original magnification). 


\section{Discussion}

The results show that the rate of cell proliferation in the rectal crypts of patients with an acute exacerbation of ulcerative proctocolitis is $65 \%$ greater than normal. Taken in association with previous evidence of increased DNA synthesis and expansion of the proliferative compartment, ${ }^{3-5}$ our data confirm that the large intestine responds to the premature shedding of its epithelial lining by generating more cells at the crypt base. Thus the turnover time of the mucosa becomes shorter in this disease.

The finding that the cell replication rate for colitis in remission is intermediate between the values obtained in normal mucosa and colitis in relapse has not previously been reported. Serafini and coworkers did not detect any significant difference in labelling index between diseased mucosa in active and quiescent phases. ${ }^{5}$

Previous studies of patients with colitis have utilised ${ }^{3} \mathrm{H}$-thymidine uptake to study cell proliferation. It is known that exogenous ${ }^{3} \mathrm{H}$-thymidine is incompletely incorporated into the DNA of intact cells, ${ }^{10}$ and variations in the duration of the $S$ phase (DNA synthesis) of the cell cycle introduce another potential error. ${ }^{11}$ These restrictions may limit the value of such methods. ${ }^{6}$ The stathmokinetic technique provides a potentially superior assessment of cell birtin rate, provided that mucosal biopsies maintained in organ culture replicate in a manner akin to intact mucosa. It is reassuring to observe that cultured biopsies show linear accumulation of metaphase figures between 16-19 hours after explantation. Pilot studies showed that no such accumulation occurred in the early culture period, probably because mitotic activity is depressed during the first few hours. ${ }^{12}$ The minimum dose of vincristine required to induce complete metaphase arrest in biopsies from patients with colitis in relapse was twice that required in normal mucosa or mucosa from colitis in remission. The reason for this discrepancy is not clear. Conceivably cell membrane permeability varies with the degree of mucosal inflammation. A similar resistance to vincristine has been reported in organ cultures of colorectal carcinoma, again with no specific explanation. ${ }^{13}$

Chronic rise of CCPR irrespective of disease activity may help to explain why some patients with ulcerative colitis develop colorectal cancer even when inflammation appears to have subsided for many years. Experimental studies with chemical carcinogens suggest that the development of neoplasia roughly parallels the intensity of hyperplasia. ${ }^{14}$ Because the greatest proliferative activity is observed in florid relapse of proctocolitis, it is hardly surprising that the extent and severity of inflammation have been implicated as prognostic factors in the development of colitis carcinoma. Perhaps a therapy that could restore CCPR completely to normal would control the malignant potential of ulcerative colitis. Measurement of CCPR in cultured biopsies offers a possible method for evaluating new therapeutic agents in this disease.

This work was supported by the Bristol and Weston Health Authority. We thank Miss Tracy Rosser and Mr T J Gradidge for technical assistance. Dr J D Davies of the University Department of Pathology at Bristol kindly carried out the histological assessments.

\section{References}

1 Shorter RG, Spencer RJ, Hallenbeck GA. Kinetic studies of the epithelial cells of the rectal mucosa in normal subjects and patients with ulcerative colitis. $G u t$ 1966; 7: 593-6.

2 Spencer RJ, Huizenga KA, Hammer CS, Shorter RG. Further studies of the kinetics of rectal epithelium in normal subjects and patients with ulcerative or granulomatous colitis. Dis Colon Rectum 1969; 12: 406-8.

3 Bleiberg $\mathrm{H}$, Mainguet $\mathrm{P}$, Galand $\mathrm{P}$, Chretien $\mathrm{J}$, Dupond-Mairesse $N$. Cell renewal in the human rectum. In vivo autoradiographic study on active ulcerative colitis. Gastroenterology 1970; 58: 851-5.

4 Eastwood GL, Trier JS. Epithelial cell renewal in cultured rectal biopsies in ulcerative colitis. Gastroenterology 1973; 64: 383-90.

5 Serafini EP, Kirk AP, Chambers TJ. Rate and pattern of epithelial cell proliferation in ulcerative colitis. Gut 1981; 22: 648-52.

6 Al-Mukhtar MYT, Polak JM, Bloom SR, Wright NA. The search for appropriate measurements of proliferative and morphological status in studies on intestinal adaptation. In: Robinson JWL, Dowling RH, Riecken E-O, eds. Mechanisms of intestinal adaptation. Lancaster: MTP Press, 1982: 3-25.

7 Truelove SC, Richards WCD. Biopsy studies in ulcerative colitis. Br Med J 1956; 1: 1315-8.

8 Allan A, Jewell DP. An in vitro model for assessment of luminal factors on rectal mucosa. Gut 1983; 24: 812-7.

9 Riddell RH, Morson BC. Value of sigmoidoscopy and biopsy in detection of carcinoma and pre-malignant change in ulcerative colitis. Gut 1979; 20: 575-80.

10 Maurer HR. Potential pitfalls of ${ }^{3} \mathrm{H}$-thymidine techniques to measure cell proliferation. Cell Tissue Kinet 1981; 14: 111-20.

11 Lewis PD. The application of cell turnover studies to neuropathology. Recent Adv Neuropathol 1979; 1: 41-5.

12 Simnet JD, Fischer JM. The relationship between cell division rate and ${ }^{3} \mathrm{H}$-thymidine incorporation in organ 
culture. J Cell Physiol 1973; 81: 171-80.

13 Pritchett CJ; Senior PV, Sunter JP, Watson AJ, Appleton DR. Wilson RG. Human colorectal tumours in short-term organ culture, a stathmokinetic study. Cell Tissue Kinet 1982; 15: 555-64.
14 Williamson RCN. Postoperative adaptation in the aetiology of intestinal cancer. In: Robinson JWL, Dowling RH, Riecken E-O, eds. Mechanisms of intestinal adaptation. Lancaster: MTP Press, 1982: 621-36. 